\title{
Effect of Dapagliflozin on Glycemic Variability in Patients with Type 2 Diabetes under Insulin Glargine Combined with Other Oral Hypoglycemic Drugs
}

\author{
Menghui Luo, Xiaocen Kong, Huiying Wang, Xiaofang Zhai, Tingting Cai, Bo Ding, Yun Hu, \\ Ting Jing, Xiaofei Su, Huiqin $\mathrm{Li} \mathbb{D}$, and Jianhua Ma
}

Department of Endocrinology, Nanjing First Hospital, Nanjing Medical University, Nanjing 210012, China

Correspondence should be addressed to Huiqin Li; lihuiqin496@126.com and Jianhua Ma; majianhua196503@126.com

Received 12 October 2020; Revised 4 November 2020; Accepted 10 November 2020; Published 24 November 2020

Academic Editor: Yoshifumi Saisho

Copyright (c) 2020 Menghui Luo et al. This is an open access article distributed under the Creative Commons Attribution License, which permits unrestricted use, distribution, and reproduction in any medium, provided the original work is properly cited.

\begin{abstract}
Aim. To evaluate the effect of an inhibitor of sodium-glucose cotransporter 2 (SGLT-2 inhibitor, dapagliflozin) on glycemic variability in type 2 diabetes mellitus (T2D) under insulin glargine combined with oral hypoglycemic drugs, using a continuous glucose monitoring system (CGMS). Methods. This prospective, self-controlled, single-center clinical trial recruited 36 patients with T2D under combined insulin glargine and oral hypoglycemic drugs. General clinical data were collected. Fasting blood glucose (FBG), postprandial blood glucose (PBG), glycosylated hemoglobin (HbA1c), and C-peptide levels were assessed before and four weeks of dapagliflozin ( $10 \mathrm{mg}$ per day) treatment. Blood glucose was monitored for 72 hours before and after treatment using CGMS. Results. After treatment with dapagliflozin, FBG decreased from $6.74 \pm 1.78$ to $5.95 \pm 1.13 \mathrm{mmol} / \mathrm{L}$ $(p<0.05)$; PBG decreased from $13.04 \pm 2.99$ to $10.92 \pm 3.26 \mathrm{mmol} / \mathrm{L}(p<0.05)$; HbAlc decreased from $7.37 \pm 0.96 \%$ to $6.94 \pm$ $0.80 \%$. The proportion of patients with $\mathrm{HbAlc}<7 \%$ increased from $27.8 \%$ to $58.3 \%$, and the proportion of patients with $\mathrm{HbA} 1 \mathrm{c}$ $<7 \%$ and without level 2 hypoglycemia increased from $27.8 \%$ to $55.6 \%(p<0.05)$. CGMS data showed reduction of the $24 \mathrm{~h}$ MBG, MAGE, time-above-range (TAR, $>10 \mathrm{mmol} / \mathrm{L}$ ), high blood glucose index (HBGI), glucose management indicator (GMI), and incremental area under the curve of the glucose level more than $10 \mathrm{mmol} / \mathrm{L}$ (AUC > 10) and an increase of time-in-range (TIR, 3.9-10 mmol/L) with treatment. Homeostasis model assessment for pancreatic beta-cell function (HOMA-beta) increased significantly with treatment $(p<0.05)$, and fewer insulin doses were required after the treatment, without increasing in hypoglycemia and urinary tract infection. Further, a stratified analysis showed that patients with higher pretreatment HbAlc and waist-to-hip ratio (WHR) had greater improvement in glycemic control. Conclusion. Dapagliflozin may reduce blood glucose levels, ameliorate glycemic variability, and improve pancreatic beta-cell function in patients with T2D under insulin glargine combined with other oral hypoglycemic drugs, especially in those with poor glucose control and abdominal obesity.
\end{abstract}

\section{Introduction}

Type 2 diabetes mellitus (T2D) is a metabolic disease characterized by chronic hyperglycemia and has become one of the common chronic diseases worldwide, especially in developing countries. In China, $10.9 \%$ of adults have diabetes, and the prediabetes prevalence rate is as high as $35.7 \%$ in China [1]. It is known that glycosylated hemoglobin (HbAlc) is closely associated with microvascular complications and cardiovascular disease outcomes [2]. More recently, evidence suggested that there are close relationships between glycemic variability and oxidative stress, endothelial dysfunction, and atherosclerosis [3-5]. Furthermore, glycemic variability is a potential risk factor for complications in patients with diabetes $[6,7]$ and is a predictor of cardiovascular complications [8]. Dapagliflozin, a selective inhibitor of sodium-glucose cotransporter 2 (SGLT-2), mainly reduces blood glucose by increasing urinary glucose excretion. Recent studies show that dapagliflozin combined with other hypoglycemic drugs with or without insulin treatment can reduce blood glucose levels and improve HbA1c levels $[9,10]$. And some studies also mentioned that SGLT-2 inhibitors can improve glycemic 
variability in T2D receiving insulin treatment [11-14]. However, it is unclear that SGLT-2 inhibitors are more effective for which groups of people. Therefore, we investigated the effect of dapagliflozin in T2D under insulin glargine combined with other oral hypoglycemic drugs using a CGMS to evaluate glucose fluctuations and find out the most suitable one.

\section{Methods}

2.1. Study Participants. This study was approved by the Ethical Committee of Nanjing First Hospital, Nanjing Medical University in Nanjing, China. Informed consent was obtained from all patients.

The inclusion criteria were as follows: (1) a diagnosis of T2D, as defined by the World Health Organization criteria published in 1999, (2) age $\geq 18$ years, (3) receiving a stable insulin glargine doses combined with oral hypoglycemic drugs for more than 3 months prior to screening, and (4) estimated glomerular filtration rate $(\mathrm{eGFR}) \geq 60 \mathrm{~mL} / \mathrm{min} / 1.73 \mathrm{~m}^{2}$. The main exclusion criteria were as follows: (1) type 1 diabetes, (2) severe hypoglycemic events or diabetic ketoacidosis (DKA) within 6 months prior to screening, (3) pregnant women, (4) cardiovascular or cerebrovascular accident $\leq 12$ weeks before screening, and (5) acute and chronic severe infectious diseases.

2.2. Study Protocol. A prospective, self-controlled, singlecenter clinical trial was conducted (NCT03631134). A total of 36 patients with T2D (21 men and 15 women) were enrolled from June 2017 to June 2019. Eligible patients received dapagliflozin (10 $\mathrm{mg}$ per day) for 4 weeks during the treatment period. Patients were asked to continue their previous treatment regimen and maintain moderate physical activity and diet. If blood glucose was less than $4.4 \mathrm{mmol} / \mathrm{L}$ by self-monitoring or the patient had symptoms of hypoglycemia, the patient was asked to reduce insulin doses. They were instructed to ingest food when blood glucose level was less than $3.9 \mathrm{mmol} / \mathrm{L}$ by self-monitoring or when they had symptoms of hypoglycemia.

Demographic and clinical data were collected by the same person throughout the study, including medical history and medicine use. Anthropometric parameters, such as height, body weight, hip circumference, and waist circumference, were measured before and after the 4-week treatment. Similarly, biochemical parameters were measured before and after treatment, including fasting blood glucose (FBG), postprandial blood glucose (PBG), alanine aminotransferase (ALT), serum creatinine (SCr), total cholesterol (TC), glycosylated hemoglobin (HbA1c), and C-peptide levels. The eGFR was calculated as follows: $\operatorname{eGFR}\left(\mathrm{mL} / \mathrm{min} / 1.73 \mathrm{~m}^{2}\right)=186 \times$ $(\mathrm{SCr} / 88.4)^{-1.154} \times(\text { age })^{-0.203} \times(0.742$ if female $)$. The homeostasis model assessment for pancreatic beta-cell function (HOMA-beta) was calculated as follows: HOMA-beta $=270$ $\times($ fasting $\mathrm{C}$ - peptide $) /[0.333 \times(\mathrm{FBG}-3.5)]$. The homeostasis model assessment of C-peptide secretion (HOMA-CR) was calculated as follows: $\mathrm{HOMA}-\mathrm{CR}=1.5+\mathrm{FBG} \times($ fasting $\mathrm{C}$ - peptide $) /(2.8 \times 0.333)$. A standard meal test was designed by a specialist researcher, which included $87.9 \%$ of carbohy- drate, $8.8 \%$ of protein, and $3.3 \%$ of fat. A CGMS (Medtronic MiniMed, USA) was used to monitor blood glucose every 5 minutes for 3 days before the study and on days 26-28. Data from 0:00 to 24:00 day 2 of CGMS was to analyze the glycemic profile. In detail, the time-in-range (TIR, 3.9-10 mmol/L), 24hour mean blood glucose (24 h MBG), 24-hour mean amplitude of glycemic excursion (MAGE), the incremental area under the curve of the glucose level (AUC), high blood glucose index (HBGI), low blood glucose index (LBGI), and glucose management indicator (GMI) were calculated.

2.3. Statistical Analysis. Statistical analyses were performed using SPSS version 20.0 (IBM Corp., Armonk, NY). Normally distributed data are presented as the mean \pm standard deviation (SD), and nonnormally distributed data are presented as median (25th-75th range). The paired $t$-test and Wilcoxon test were used to evaluate differences in glycemic profile before and after treatment. The independentsamples $t$-test and Mann-Whitney $U$ test were used to evaluate differences in glycemic profile between patients who did and did not achieve HbAlc $\geq 7 \%$ with treatment. The oneway analysis of variance (ANOVA) and $K$-independent samples test were used to evaluate differences in pretreatment characteristics among tertiles of the treatment-related reduction in the HbA1c level. The chi-square test was used to compare qualitative data. The accepted level of significance was 0.05 , using two-tailed tests.

\section{Results}

3.1. Baseline Characteristics. The baseline characteristics of the participants are shown in Table 1. The average duration of diabetes was $10.92 \pm 4.92$ years, and the average pretreatment HbA1c level was $7.37 \pm 0.96 \%$. The pretreatment $\mathrm{HbA} 1 \mathrm{c}$ level was higher than $7 \%$ in $72.2 \%$ of patients.

3.2. The 24 h Glycemic Profile. The $24 \mathrm{~h}$ CGMS glucose profile of patients is shown in Figure 1. The $24 \mathrm{~h} \mathrm{MBG,} \mathrm{MAGE,}$ HBGI, GMI, the incremental area under the curve of the glucose level more than $10 \mathrm{mmol} / \mathrm{L}$ (AUC > 10), and timeabove-range (TAR, $>10 \mathrm{mmol} / \mathrm{L}$ ) were reduced, and the TIR was increased, after the 4-week treatment compared to pretreatment values $(p<0.05)$. However, there was no difference in the time-below-range (TBR, $<3.9 \mathrm{mmol} / \mathrm{L}$ ) (Table 2).

Additionally, patients who achieved $\mathrm{HbAlc} \geq 7 \%$ after treatment showed obvious improvement in $24 \mathrm{~h} \mathrm{MBG}$, TAR, AUC3.9-10, HBGI, GMI, and AUC > $10 \quad(p<0.05)$ (Table 3).

\subsection{Glycemic Control and Pancreatic Beta-Cell Function.} Compared to pretreatment values, patients required fewer daily insulin doses after treatment for a 4 -week treatment period $(p<0.05)$. The homeostasis model assessment for pancreatic beta-cell function (HOMA-beta) increased significantly after treatment. Additionally, FBG, PBG, and HbAlc levels after the standard meal test were lower after treatment compared to pretreatment values (Table 4). The proportion of patients who achieved HbAlc $<7 \%$ increased from $27.8 \%$ to $58.3 \%(p<0.05)$. Additionally, the proportion of patients who achieved $\mathrm{HbAlc}<7 \%$ without level 2 
TABle 1: Patient baseline characteristics.

\begin{tabular}{lc}
\hline Characteristics & Whole group $(n=36)$ \\
\hline Age (years) & $58.33 \pm 9.50$ \\
Sex (M/F) & $21 / 15$ \\
Diabetes duration (years) & $10.92 \pm 4.92$ \\
Insulin dose (IU/d) & $21.67 \pm 7.89$ \\
BMI (kg/m ${ }^{2}$ ) & $25.22 \pm 3.21$ \\
Body weight (kg) & $69.28 \pm 11.43$ \\
Waist-to-hip radio & $0.94 \pm 0.05$ \\
SBP (mmHg) & $135.86 \pm 15.23$ \\
DBP (mmHg) & $84.47 \pm 20.68$ \\
FBG (mmol/L) & $6.74 \pm 1.78$ \\
HbA1c (\%) & $7.37 \pm 0.96$ \\
ALT (U/L) & $24.53 \pm 12.14$ \\
eGFR (mL/min/1.73 ${ }^{2}$ ) & $111.03 \pm 19.59$ \\
TC (mmol/L) & $4.51 \pm 1.03$ \\
HOMA-beta & $317.64 \pm 241.91$ \\
HOMA-CR & $9.89 \pm 7.60$ \\
Oral hypoglycemic drugs (\%) & \\
Secretagogues & 50.0 \\
Metformin & 77.8 \\
Thiazolidine & 8.3 \\
$\alpha$-Glucosidase inhibitor & 52.8 \\
DPP4 inhibitor & (m) \\
\hline & \\
\hline
\end{tabular}

BMI: body mass index; SBP: systolic blood pressure; DBP: diastolic blood pressure; FBG: fasting blood glucose; HbAlc: glycosylated hemoglobin; ALT: alanine aminotransferase; eGFR: estimated glomerular filtration rate; TC: total cholesterol; HOMA-beta: homeostasis model assessment for pancreatic beta-cell function; HOMA-CR: homeostasis model assessment of C-peptide secretion; DPP4: dipeptidase-4 inhibitor.

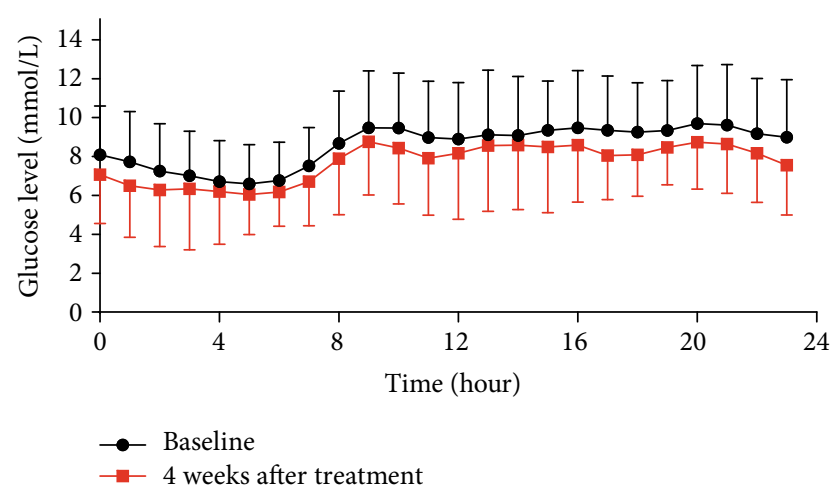

FIgURE 1: 24 h CGMS glucose profile of patients.

hypoglycemia (glucose concentration $<3.0 \mathrm{mmol} / \mathrm{L}$ ) increased from $27.8 \%$ to $55.6 \%(p<0.05)$.

In order to further explore the characteristics of patients with a greater decrease in the HbAlc level, the baseline data were analyzed in the terms of tertiles in the treatment-related reduction in the HbAlc level. High, mid, and low degree of treatment-related reduction in the HbAlc level were defined as reductions $\geq 0.6 \%$, 0.3 $0.5 \%$, and $\leq 0.2 \%$, respectively. We found that patients with a higher pretreatment waist-to-hip ratio and $\mathrm{HbA1c}$ level had a greater treatment-related decrease in the HbAlc level (Table 5).

3.4. Hypoglycemia and Urinary Tract Infection. Level 3 hypoglycemia (defined as a severe event characterized by altered mental and/or physical status requiring assistance for treatment of hypoglycemia) did not occur during the study period. There was no change in the rates of level 1 hypoglycemia (glucose concentration $<3.9 \mathrm{mmol} / \mathrm{L}$ and $\geq 3.0 \mathrm{mmol} / \mathrm{L}$ ) and level 2 hypoglycemia (glucose concentration $<3.0$ $\mathrm{mmol} / \mathrm{L})$ with treatment $(p>0.05)$. Furthermore, the incidence of urinary tract infection did not change with treatment.

\section{Discussion}

The present study showed that patients under insulin glargine combined with oral hypoglycemic drugs benefited from dapagliflozin treatment in terms of improvements in the mean glucose level and glycemic variability, without an increase in the incidence of hypoglycemia and urinary tract infection. Patients with higher HbAlc and waist-tohip ratio before treatment were more beneficial in improving $\mathrm{HbAlc}$. Another important outcome from the present study was that the SGLT-2 inhibitor, dapagliflozin, was able to partially replace exogenous insulin and improve pancreatic beta-cell function.

The HbAlc is used to assess glycemic control in diabetes mellitus during three months as a gold standard. Many large studies showed a higher mean HbAlc level usually companies with a higher incidence of diabetic complications, even can predict and partly explain cardiovascular disease [15-17]. However, the HbAlc level is affected by individual factors, including genetic, hematological conditions, and ethnicity [18-20]. More importantly, the HbAlc cannot reflect glycemic variability. It was reported that glycemic variability still is an independent risk of diabetic neuropathy, although at the same mean glucose level [21]. And glycemic variability has a greater and worse effect on cardiovascular disease than HbAlc [22]. Thus, It was not enough to rely only on $\mathrm{HbAlc}$ to guide glycemic management. Recently, some studies have focused on glycemic variability as another metric for glycemic control. The relationship between diabetes-related complications and glycemic variability may result from cardiovascular damage and hypoglycemia [23]. CGMSs utilize a monitoring technology that indirectly detects the blood glucose level in the interstitial fluid via a sensor every 5 minutes. This provides a complete and available glycemic profile and enables us to a better understanding of the glycemic variability and detection of hypoglycemia in time.

The American Diabetes Association emphasizes the importance of SGLT-2 inhibitors as combination therapy, especially in patients with poor glucose control and 
TABLE 2: $24 \mathrm{~h}$ glycemic profiles.

\begin{tabular}{lccc}
\hline & Baseline & 4 weeks after the treatment & $p$ \\
\hline 24 h MBG $(\mathrm{mmol} / \mathrm{L})$ & $8.56 \pm 1.77$ & $7.43 \pm 1.03^{*}$ & 0.001 \\
MAGE $(\mathrm{mmol} / \mathrm{L})$ & $4.80 \pm 2.73$ & $3.73 \pm 2.10^{*}$ & 0.048 \\
TIR $(\%)$ & $72.45 \pm 26.19$ & $85.56 \pm 14.88^{*}$ & 0.002 \\
TAR (\%) & $20.66(6.95,40.80)$ & $9.38(0.00,18.49)^{*}$ & 0.001 \\
TBR $(\%)$ & $0.00(0.00,0.00)$ & $0.00(0.00,1.39)$ & 0.796 \\
AUC3.9-10 $(\mathrm{mmol} / \mathrm{L} * \mathrm{~d})$ & $242.04 \pm 61.11$ & $199.14 \pm 48.15^{*}$ & 0.002 \\
AUC $>10(\mathrm{mmol} / \mathrm{L} * \mathrm{~d})$ & $11.35(2.64,51.54)$ & $5.78(0.00,20.51)^{*}$ & 0.003 \\
AUC $<3.9(\mathrm{mmol} / \mathrm{L} * \mathrm{~d})$ & $0.00(0.00,0.00)$ & $0.00(0.00,0.00)$ & 0.959 \\
HBGL & $6.08 \pm 5.38$ & $4.04 \pm 2.83^{*}$ & 0.000 \\
LBGL & $0.68(0.00,2.23)$ & $1.18(0.25,2.68)$ & 0.223 \\
GMI $(\mathrm{mmol} / \mathrm{mol})$ & $52.99 \pm 8.32$ & $47.68 \pm 4.88^{*}$ & 0.001 \\
\hline
\end{tabular}

Data were shown as mean \pm SD or median (first quartile, third quartile). * Baseline vs. 4 weeks after the treatment: $p<0.05 .24$ h MBG: 24 -hour mean blood glucose; MAGE: 24-hour mean amplitude of glycemic excursion; TIR: time-in-range (3.9-10 mmol/L); TAR: time-above-target range ( $>10 \mathrm{mmol} / \mathrm{L})$; TBR: time-below-target ranges $(<3.9 \mathrm{mmol} / \mathrm{L})$; AUC3.9-10: the incremental area under the curve of the glucose level between 3.9 and $10 \mathrm{mmol} / \mathrm{L}$; AUC > 10: the incremental area under the curve of the glucose level more than $10 \mathrm{mmol} / \mathrm{L} ; \mathrm{AUC}<3.9$ : the incremental area under the curve of the glucose level less than 3.9 mmol/L; HBGI: high blood glucose index; LBGI: low blood glucose index; GMI: glucose management indicator.

TABLE 3: Dynamic blood glucose profile of patients with different HbAlc stratification before and after treatment.

\begin{tabular}{|c|c|c|c|c|c|c|}
\hline & \multicolumn{2}{|c|}{ Baseline } & \multicolumn{2}{|c|}{4 weeks after treatment } & \multicolumn{2}{|c|}{$\Delta$} \\
\hline & High- $A_{1 C}(\geq 7 \%)$ & Low- $\mathrm{A}_{1 \mathrm{C}}(<7 \%)$ & High- $A_{1 C}(\geq 7 \%)$ & Low- $\mathrm{A}_{1 \mathrm{C}}(<7 \%)$ & High- $A_{1 C}(\geq 7 \%)$ & Low-A $\mathrm{A}_{1 \mathrm{C}}(<7 \%)$ \\
\hline $24 \mathrm{~h} \mathrm{MBG}$ & $9.07 \pm 1.72$ & $7.23 \pm 1.09^{*}$ & $7.52 \pm 1.10$ & $7.21 \pm 0.88$ & $-1.55 \pm 1.76$ & $-0.02 \pm 1.48^{*}$ \\
\hline MAGE & $5.60 \pm 2.66$ & $2.71 \pm 1.67^{*}$ & $3.97 \pm 2.16$ & $3.11 \pm 1.87$ & $-1.63 \pm 3.17$ & $0.39 \pm 2.66$ \\
\hline TIR (\%) & $65.10 \pm 26.35$ & $91.56 \pm 13.32^{* *}$ & $82.32 \pm 15.71$ & $93.99 \pm 8.12^{*}$ & $17.22 \pm 24.27$ & $2.43 \pm 12.95$ \\
\hline TAR (\%) & $24.83(12.76,43.15)$ & $0.00(0.00,16.93)^{*}$ & $9.72(0.00,25.70)$ & $0.70(0.00,12.76)$ & $-17.54(-29.08,-3.38)$ & $0.52(-4.17,3.22)^{*}$ \\
\hline TBR (\%) & $0.00(0.00,1.74)$ & $0.00(0.00,0.00)$ & $0.00(0.00,1.65)$ & $0.00(0.00,0.35)$ & $0.00(-1.74,1.39)$ & $0.00(0.00,0.35)$ \\
\hline AUC3.9-10 & $260.22 \pm 51.92$ & $194.75 \pm 59.95^{*}$ & $200.91 \pm 48.03$ & $194.54 \pm 50.77$ & $-59.32 \pm 69.79$ & $-0.21 \pm 83.29^{*}$ \\
\hline AUC $>10$ & $26.48(5.41,70.53)$ & $0.00(0.00,9.32)^{* *}$ & $6.89(0.00,28.58)$ & $0.10(0.00,7.03)$ & $-12.37(-52.96,0.15)$ & $0.00(-6.57,5.20)^{*}$ \\
\hline AUC $<3.9$ & $0.00(0.00,0.47)$ & $0.00(0.00,0.00)$ & $0.00(0.00,0.23)$ & $0.00(0.00,0.02)$ & $0.00(-0.47,0.11)$ & $0.00(0.00,0.02)$ \\
\hline HBGI & $8.33 \pm 5.42$ & $2.84 \pm 2.59^{* *}$ & $4.57 \pm 3.00$ & $2.64 \pm 1.79$ & $-3.75 \pm 4.13$ & $-0.20 \pm 2.68^{*}$ \\
\hline LBGI & $0.45(0.01,3.03)$ & $0.89(0.00,1.57)$ & $1.30(0.39,3.24)$ & $0.51(0.19,1.64)$ & $0.78 \pm 3.42$ & $-0.05 \pm 1.58$ \\
\hline GMI & $55.41 \pm 8.10$ & $46.72 \pm 5.13^{* *}$ & $48.10 \pm 5.15$ & $46.61 \pm 4.14$ & $-7.31 \pm 8.28$ & $-0.11 \pm 6.98^{*}$ \\
\hline
\end{tabular}

${ }^{*} p<0.05 ;{ }^{* *} p<0.01 .24$ h MBG: 24-hour mean blood glucose; MAGE: 24-hour mean amplitude of glycemic excursion; TIR: time-in-range (3.9-10 mmol/L); TAR: time-above-target range $(>10 \mathrm{mmol} / \mathrm{L})$; TBR: time-below-target ranges $(<3.9 \mathrm{mmol} / \mathrm{L})$; AUC3.9-10: the incremental area under the curve of the glucose level between 3.9 and $10 \mathrm{mmol} / \mathrm{L}$; AUC > 10: the incremental area under the curve of the glucose level more than 10 mmol/L; AUC < 3.9: the incremental area under the curve of the glucose level less than $3.9 \mathrm{mmol} / \mathrm{L}$; HBGI: high blood glucose index; LBGI: low blood glucose index; GMI: glucose management indicator.

atherosclerotic cardiovascular disease [24]. It was reported that HbA1c and FPG levels showed greater reductions with 52 weeks of dapagliflozin treatment, compared to that with placebo [25]. Similarly, our study suggested that FBG, PBG, and $\mathrm{HbA} 1 \mathrm{c}$ reduced with dapagliflozin treatment. Further, we found that patients with abdominal obesity and poor glycemic control might be more suitable for dapagliflozin treatment.

A previous study showed that dapagliflozin had a beneficial effect on glycemic variability in T2D under either insulin or metformin [26]. Another study indicated that dapagliflozin also has a positive effect on glycemic control, without the occurrence of hypoglycemia, in patients with type 1 diabetes [27]. This study explored that dapagliflozin was used as monotherapy or as a supplement to other oral hypoglycemic drugs or insulin for diabetic treatment. Our study also showed dapagliflozin improved glycemic variability without increasing the incidence of hypoglycemia. Furthermore, we analyzed the differences in the glycemic profile of patients with different HbAlc levels, in order to find out that dapagliflozin was more suitable for which kind of population in T2D under insulin glargine combined with other oral hypoglycemic drugs. As a result, using CGM data, we found that patients with higher HbAlc levels showed 
TABLE 4: Glycemic control and pancreatic $\beta$-cell function.

\begin{tabular}{lccc}
\hline & Baseline & 4 weeks after treatment & $p$ \\
Insulin dose (IU/d) & $21.67 \pm 7.89$ & $18.28 \pm 7.52^{*}$ & 0.000 \\
HbA1c (\%) & $7.37 \pm 0.96$ & $6.94 \pm 0.80^{*}$ & 0.000 \\
FBG (mmol/L) & $6.74 \pm 1.78$ & $5.95 \pm 1.13^{*}$ & 0.015 \\
PBG 30 min (mmol/L) & $8.59 \pm 1.85$ & $7.59 \pm 1.83^{*}$ & 0.004 \\
PBG 120 min (mmol/L) & $13.04 \pm 2.99$ & $10.92 \pm 3.26$ & 0.005 \\
C-peptide 0 min (ng/mL) & $1.10 \pm 0.88$ & $1.15 \pm 0.73$ & 0.589 \\
C-peptide 30 min (ng/mL) & $1.62 \pm 1.04$ & $1.55 \pm 0.87$ & 0.372 \\
C-peptide 120 min (ng/mL) & $3.24 \pm 2.08$ & $3.51 \pm 1.66$ & 0.382 \\
HOMA-CR & $9.89 \pm 7.60$ & $9.30 \pm 5.46$ & 0.543 \\
HOMA-beta & $317.64 \pm 241.91$ & $412.51 \pm 273.48^{*}$ & 0.046 \\
\hline
\end{tabular}

Data were shown as mean \pm SD or median (first quartile, third quartile). ${ }^{*}$ Baseline vs. 4 weeks after the treatment: $p<0.05$. HbAlc: glycosylated hemoglobin; FBG: fasting blood glucose; PBG 30 min: postprandial blood glucose at $30 \mathrm{~min}$ after a standard meal; PBG 120 min: postprandial blood glucose at 120 min after a standard meal; C-peptide $0 \mathrm{~min}$ : C-peptide level at $0 \mathrm{~min}$ after a standard meal; C-peptide $30 \mathrm{~min}$ : C-peptide level at $30 \mathrm{~min}$ after a standard meal; C-peptide 120 min: C-peptide level at 120 min after a standard meal; HOMA-CR: homeostasis model assessment of C-peptide secretion; HOMA-beta: homeostasis model assessment for pancreatic beta-cell.

TABle 5: Difference in baseline characteristics between different drops of HbAlc.

\begin{tabular}{|c|c|c|c|c|}
\hline & High degree $(\geq 0.6 \%)$ & Mid degree $(0.3-0.5 \%)$ & Low degree $(\leq 0.2 \%)$ & $p$ \\
\hline Age (years) & $59.42 \pm 8.40$ & $52.67 \pm 10.84$ & $60.87 \pm 8.63$ & 0.108 \\
\hline Duration (years) & $11.75 \pm 4.75$ & $11.33 \pm 5.52$ & $10.00 \pm 4.87$ & 0.641 \\
\hline Body weight (kg) & $70.04 \pm 10.04$ & $73.17 \pm 9.41$ & $66.33 \pm 13.31$ & 0.362 \\
\hline BMI $\left(\mathrm{kg} / \mathrm{m}^{2}\right)$ & $25.60 \pm 3.50$ & $26.23 \pm 2.84$ & $24.31 \pm 3.14$ & 0.331 \\
\hline Waist-to-hip ratio & $0.96 \pm 0.04$ & $0.94 \pm 0.04$ & $0.91 \pm 0.06$ & 0.025 \\
\hline $\mathrm{eGFR}\left(\mathrm{mL} / \mathrm{min}^{-1} / 1.73 \mathrm{~m}^{2}\right)$ & $121.69 \pm 10.64$ & $107.47 \pm 15.10$ & $104.63 \pm 24.31$ & 0.086 \\
\hline HbAlc (\%) & $7.94 \pm 1.19$ & $7.32 \pm 0.79$ & $6.93 \pm 0.58$ & 0.029 \\
\hline Insulin dose (IU/d) & $22.17 \pm 8.80$ & $24.44 \pm 10.28$ & $19.60 \pm 4.95$ & 0.606 \\
\hline HOMA-CR & $8.40 \pm 3.89$ & $11.32 \pm 5.06$ & $10.15 \pm 10.72$ & 0.723 \\
\hline HOMA-beta & $289.91 \pm 224.45$ & $265.75 \pm 133.65$ & $370.91 \pm 304.27$ & 0.584 \\
\hline
\end{tabular}

Data were shown as mean \pm SD or median (first quartile, third quartile). BMI: body mass index; eGFR: estimated glomerular filtration rate; HbAlc: glycosylated hemoglobin; HOMA-CR: homeostasis model assessment of C-peptide secretion; HOMA-beta: homeostasis model assessment for pancreatic beta-cell.

shorter duration of euglycemia, longer duration of hyperglycemia, greater glycemic variability, and greater improvement in hyperglycemia and euglycemia than patients with lower levels of HbAlc.

SGLT2 inhibitors can increase insulin secretion and betacell mass but cannot increase insulin sensitivity [28-30]. However, $\mathrm{Xu}$ el. [31] reported that mice who were fed a high-fat diet showed amelioration of insulin resistance, via an increase in insulin receptors, after SGLT-2 inhibitor treatment. However, there are few studies to evaluate the effect of SGLT-2 inhibitors combined with insulin glargine and other oral hypoglycemic drugs on pancreatic beta-cell in T2D. We focused on dapagliflozin as a basal insulin supplement therapy with T2D and showed that dapagliflozin played an important role in improving pancreatic beta-cells and reducing insulin doses.
The present study has some limitations. Its sample size was small. In the future, we intend to increase the number of samples to further confirm our conclusions. And the study period was short; therefore, it is unclear whether glycemic variability improved by dapagliflozin can contribute to long-term benefit from T2D under insulin glargine combined with other oral hypoglycemic drugs. We need to extend the research time to confirm it. Our study has no control group; it may be difficult to exclude the placebo effect of patients after taking medicine.

In summary, patients with $\mathrm{T} 2 \mathrm{D}$ under insulin glargine combined with oral hypoglycemic drugs had lower HbA1c levels and glycemic variability and better beta-cell function, consistent with a reduced need for insulin doses, especially in patients with higher pretreatment HbAlc levels, after dapagliflozin treatment. More importantly, patients with 
poor glucose control and abdominal obesity had greater benefits with dapagliflozin treatment.

\section{Data Availability}

All the data used to support the findings of this study are available from the corresponding author upon request.

\section{Conflicts of Interest}

All authors accepted the final manuscript and had no conflicts of interest.

\section{Authors' Contributions}

JHM, HQL, and XFS contributed to the design of the study. YH, TJ, TTC, and BD contributed to the conduct/data collection. HYW and XFZ contributed to data analysis. MHL, $\mathrm{HQL}$, and XCK contributed to the final manuscript writing. Menghui Luo and Xiaocen Kong are co-first authors and equal contributors.

\section{Acknowledgments}

All authors appreciated the patients of the study. The study was supported by the National Key R\&D Program of China (No. 2018YFC1314102).

\section{References}

[1] L. Wang, P. Gao, M. Zhang et al., "Prevalence and ethnic pattern of diabetes and prediabetes in China in 2013," JAMA, vol. 317, no. 24, pp. 2515-2523, 2017.

[2] A. American Diabetes, "6. Glycemic targets: standards of medical care in diabetes-2020," Diabetes Care, vol. 43, Supplement 1, pp. S66-S76, 2019.

[3] K. Torimoto, Y. Okada, H. Mori, and Y. Tanaka, "Relationship between fluctuations in glucose levels measured by continuous glucose monitoring and vascular endothelial dysfunction in type 2 diabetes mellitus," Cardiovasc Diabetol, vol. 12, no. 1, p. 1, 2013.

[4] S. Costantino, F. Paneni, R. Battista et al., "Impact of glycemic variability on chromatin remodeling, oxidative stress, and endothelial dysfunction in patients with type 2 diabetes and with target HbA1c levels," Diabetes, vol. 66, no. 9, pp. 24722482, 2017.

[5] G. Su, S. Mi, H. Tao et al., "Association of glycemic variability and the presence and severity of coronary artery disease in patients with type 2 diabetes," Cardiovasc Diabetol, vol. 10, no. 1, p. 19, 2011.

[6] Y. M. Hu, L. H. Zhao, X. L. Zhang et al., "Association of glycaemic variability evaluated by continuous glucose monitoring with diabetic peripheral neuropathy in type 2 diabetic patients," Endocrine, vol. 60, no. 2, pp. 292-300, 2018.

[7] L. Monnier, C. Colette, J. L. Schlienger, B. Bauduceau, and D. R Owens, "Glucocentric risk factors for macrovascular complications in diabetes: glucose 'legacy' and 'variability'-what we see, know and try to comprehend," Diabetes \& Metabolism, vol. 45, no. 5, pp. 401-408, 2019.

[8] X. Wang, X. Zhao, T. Dorje, H. Yan, J. Qian, and J. Ge, "Glycemic variability predicts cardiovascular complications in acute myocardial infarction patients with type 2 diabetes mellitus," International Journal of Cardiology, vol. 172, no. 2, pp. 498-500, 2014.

[9] S. Matthaei, K. Bowering, K. Rohwedder, A. Grohl, S. Parikh, and Study 05 Group, "Dapagliflozin improves glycemic control and reduces body weight as add-on therapy to metformin plus sulfonylurea: a 24-week randomized, double-blind clinical trial," Diabetes Care, vol. 38, no. 3, pp. 365-372, 2015.

[10] K. Kostev, S. Pscherer, R. Rist, S. Busch, and M. F. Scheerer, "Changes in glycemic control and body weight after initiation of dapagliflozin or basal insulin supported oral therapy in type 2 diabetes: a primary care database study," Journal of Diabetes Science and Technology, vol. 11, no. 3, pp. 590-596, 2016.

[11] M. Matsumura, Y. Nakatani, S. Tanka et al., "Efficacy of additional canagliflozin administration to type 2 diabetes patients receiving insulin therapy: examination of diurnal glycemic patterns using continuous glucose monitoring (CGM)," Diabetes Therapy, vol. 8, no. 4, pp. 821-827, 2017.

[12] S. H. LEE, K. W. Min, B. W. Lee et al., "Effect of dapagliflozin as an add-on therapy to insulin on the glycemic variability in subjects with type 2 diabetes mellitus (DIVE): a multicenter, placebo-controlled, double-blind, randomized study," Diabetes \& Metabolism Journal, 2020.

[13] K. Torimoto, Y. Okada, Y. Goshima, A. Tokutsu, Y. Sato, and Y. Tanaka, "Addition of canagliflozin to insulin improves glycaemic control and reduces insulin dose in patients with type 2 diabetes mellitus: a randomized controlled trial," Diabetes, Obesity \& Metabolism, vol. 21, no. 9, pp. 2174-2179, 2019

[14] S. Takeishi, H. Tsuboi, and S. Takekoshi, "Comparison of tofogliflozin $20 \mathrm{mg}$ and ipragliflozin $50 \mathrm{mg}$ used together with insulin glargine $300 \mathrm{U} / \mathrm{mL}$ using continuous glucose monitoring (CGM): a randomized crossover study," Endocrine Journal, vol. 64, no. 10, pp. 995-1005, 2017.

[15] R. G. Miller, T. Costacou, and T. J. Orchard, "Risk factor modeling for cardiovascular disease in type 1 diabetes in the Pittsburgh Epidemiology of Diabetes Complications (EDC) study: a comparison with the diabetes control and complications trial/epidemiology of diabetes interventions and complications study (DCCT/EDIC)," Diabetes, vol. 68, no. 2, pp. 409419, 2019.

[16] I. M. Stratton, A. I. Adler, H. A. Neil et al., "Association of glycaemia with macrovascular and microvascular complications of type 2 diabetes (UKPDS 35): prospective observational study," BMJ, vol. 321, no. 7258, pp. 405-412, 2000.

[17] the DCCT/EDIC Research Group, I. Bebu, B. H. Braffett et al., "The relationship of blood glucose with cardiovascular disease is mediated over time by traditional risk factors in type 1 diabetes: the DCCT/EDIC study," Diabetologia, vol. 60, no. 10, pp. 2084-2091, 2017.

[18] R. A. Booth, Y. Jiang, H. Morrison, H. Orpana, S. Rogers Van Katwyk, and C. Lemieux, "Ethnic dependent differences in diagnostic accuracy of glycated hemoglobin (HbAlc) in Canadian adults," Diabetes Research and Clinical Practice, vol. 136, pp. 143-149, 2018.

[19] N. Soranzo, S. Sanna, E. Wheeler et al., "Common variants at 10 genomic loci influence hemoglobin A1C levels via glycemic and nonglycemic pathways," Diabetes, vol. 59, no. 12, pp. 3229-3239, 2010.

[20] Z. T. Bloomgarden, S. E. Inzucchi, E. Karnieli, and D. Le Roith, "The proposed terminology 'A(1c)-derived average glucose' is inherently imprecise and should not be adopted," Diabetologia, vol. 51, no. 7, pp. 1111-1114, 2008. 
[21] J. E. Jun, S. E. Lee, Y. B. Lee et al., "Continuous glucose monitoring defined glucose variability is associated with cardiovascular autonomic neuropathy in type 1 diabetes," Diabetes/Metabolism Research and Reviews, vol. 35, no. 2, p. e3092, 2019.

[22] G. Su, S. H. Mi, H. Tao et al., "Impact of admission glycemic variability, glucose, and glycosylated hemoglobin on major adverse cardiac events after acute myocardial infarction," Diabetes Care, vol. 36, no. 4, pp. 1026-1032, 2013.

[23] A. Ceriello, L. Monnier, and D. Owens, "Glycaemic variability in diabetes: clinical and therapeutic implications," The Lancet Diabetes \& Endocrinology, vol. 7, no. 3, pp. 221-230, 2019.

[24] American Diabetes Association, "9. Pharmacologic approaches to glycemic treatment: standards of medical care in diabetes2020," Diabetes Care, vol. 43, Supplement 1, pp. S98-S110, 2020.

[25] K. B. S. Matthaei, K. Bowering, K. Rohwedder et al., "Durability and tolerability of dapagliflozin over 52 weeks as add-on to metformin and sulphonylurea in type 2 diabetes," Diabetes, Obesity and Metabolism, vol. 17, no. 11, pp. 1075-1084, 2015.

[26] R. R. Henry, P. Strange, R. Zhou et al., "Effects of dapagliflozin on 24-hour glycemic control in patients with type 2 diabetes: a randomized controlled trial," Diabetes Technology \& Therapeutics, vol. 20, no. 11, pp. 715-724, 2018.

[27] P. Dandona, C. Mathieu, M. Phillip et al., "Efficacy and safety of dapagliflozin in patients with inadequately controlled type 1 diabetes (DEPICT-1): 24 week results from a multicentre, double-blind, phase 3, randomised controlled trial," The Lancet Diabetes \& Endocrinology, vol. 5, no. 11, pp. 864-876, 2017.

[28] E. Ferrannini, E. Muscelli, S. Frascerra et al., "Metabolic response to sodium-glucose cotransporter 2 inhibition in type 2 diabetic patients," The Journal of Clinical Investigation, vol. 124, no. 2, pp. 499-508, 2014.

[29] J. Shirakawa, K. Tajima, T. Okuyama et al., "Luseogliflozin increases beta cell proliferation through humoral factors that activate an insulin receptor- and IGF-1 receptor-independent pathway," Diabetologia, vol. 63, no. 3, pp. 577-587, 2020.

[30] K. Takahashi, A. Nakamura, H. Miyoshi et al., "Effect of the sodium-glucose cotransporter 2 inhibitor luseogliflozin on pancreatic beta cell mass in $\mathrm{db} / \mathrm{db}$ mice of different ages," Scientific Reports, vol. 8, no. 1, p. 6864, 2018.

[31] L. Xu, N. Nagata, M. Nagashimada et al., "SGLT2 inhibition by empagliflozin promotes fat utilization and browning and attenuates inflammation and insulin resistance by polarizing M2 macrophages in diet-induced obese mice," EBioMedicine, vol. 20, pp. 137-149, 2017. 